\title{
Stimuli-Responsive Chemomechanical Actuation: A Hybrid Materials Approach
}

\section{Citation}

Zarsar, Lauren D., and Joanna Aizenberg. 2014. Stimuli-Responsive Chemomechanical

Actuation: A Hybrid Materials Approach. Acc. Chem. Res. 47, no. 2: 530-539. D0I: 10.1021/ $\operatorname{ar} 4001923$

\section{Published Version}

doi:10.1021/ar4001923

\section{Permanent link}

http://nrs.harvard.edu/urn-3:HUL.InstRepos:27663228

\section{Terms of Use}

This article was downloaded from Harvard University's DASH repository, and is made available under the terms and conditions applicable to Open Access Policy Articles, as set forth at http:// nrs.harvard.edu/urn-3:HUL.InstRepos:dash.current.terms-of-use\#OAP

\section{Share Your Story}

The Harvard community has made this article openly available.

Please share how this access benefits you. Submit a story.

Accessibility 


\title{
Stimuli-Responsive Chemo-Mechanical Actuation: A Hybrid Materials Approach
}

\author{
Lauren D. Zarzar ${ }^{\dagger}$ and Joanna Aizenberg ${ }^{\dagger+\beta^{*}}$ \\ ${ }^{\dagger}$ Department of Chemistry and Chemical Biology, Harvard University, Cambridge, \\ Massachusetts 02138, United States \\ ${ }^{\ddagger}$ School of Engineering and Applied Sciences, Harvard University, Cambridge, \\ Massachusetts 02138, United States \\ ${ }^{\S}$ Wyss Institute for Biologically Inspired Engineering, Harvard University, Boston, \\ Massachusetts 02115, United States
}

\section{Conspectus}

Dynamic materials which can sense changes in their surroundings and functionally respond by autonomously altering their shape, surface chemistry, transparency, color, wetting behavior, adhesiveness, etc. are primed to be integral components of future "smart" technologies. Indeed, a fundamental reason for the adaptability of biological organisms is their innate ability to so elegantly convert environmental or chemical cues into mechanical motion/reconfiguration on both the molecular and macroscale. However, design and engineering of robust chemomechanical behavior in artificial materials has proven a challenge; such systems can be quite complex and often require intricate coordination between both chemical and mechanical inputs/outputs as well as the combination of multiple materials working cooperatively to achieve the proper functionality. It's critical to not only understand the fundamental behaviors of existing dynamic chemo-mechanical systems, but also to apply that knowledge and explore new avenues for design of novel materials platforms which could provide a basis for future adaptive 
technologies. In this Account, we explore the chemo-mechanical behavior, properties, and applications of hybrid-material surfaces consisting of environmentally-sensitive hydrogels integrated within arrays of high-aspect-ratio nano/microstructures. This bio-inspired approach, in which the volume-changing hydrogel acts as the "muscle" that reversibly actuates the microstructured "bone", is highly tunable and customizable; although straightforward in concept, the combination of just these two materials (structures and hydrogel) has given rise to a far more complex set of actuation mechanisms and behaviors. Variations in how the hydrogel is physically integrated within the structure array provide the basis for three fundamental mechanisms of actuation, each with its own set of responsive properties and chemo-mechanical behavior. Further control over how the chemical stimulus is applied to the surface, such as with microfluidics, allows for generation of more precise and varied patterns of actuation. We also discuss the possible applications of these hybrid surfaces for chemo-mechanical manipulation of reactions, including the generation of chemo-mechanical feedback loops. Comparing and contrasting these many approaches and techniques, we aim to put into perspective their highly tunable and diverse capabilities but also their future challenges and impacts.

Conspectus graphic:

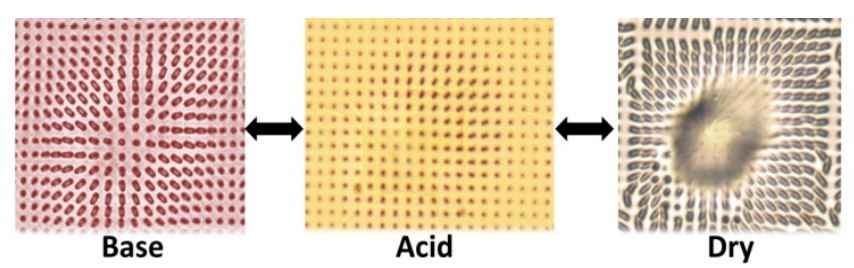

\section{Biographical Information}

Lauren D. Zarzar received a B.A. (Chemistry) and B.S. (Economics) from the University of Pennsylvania School of Arts and Sciences and Wharton School respectively and a Ph.D. from Harvard University. She was a recipient of the NSF Graduate Research Fellowship and the DoD National Defense Science and Engineering Graduate Fellowship.

Joanna Aizenberg is the Amy Smith Berylson Professor of Materials Science and the Director of the Kavli Institute at Harvard University. She pursues a broad range of research interests that include biomimetics, self-assembly, smart materials, crystal engineering, surface science, and biooptics. Aizenberg's select awards include: R\&D 100 Awards for best innovations in 2012 and 
2013, Fred Kavli Distinguished Lectureship in Nanoscience, MRS 2009; and Ronald Breslow Award for the Achievement in Biomimetic Chemistry, ACS 2008. Aizenberg is an AAAS and APS Fellow; she has served on the Board of Directors of the Materials Research Society and Board on Physics and Astronomy of the National Academies.

\section{Introduction}

Design of synthetic systems that sense and respond to environmental stimuli and chemical signals on both a microscopic and macroscopic level has been a longstanding scientific pursuit. ${ }^{1}$ But from a biological perspective, the interplay between signaling molecules and/or external stimuli and mechanical reconfiguration is commonplace, indeed fundamental, to nature's ability to self-regulate and adapt. From the nanoscale (motor-like action of ATPases, ${ }^{2}$ conformational changes of proteins allowing allosteric regulation ${ }^{3}$ ) to the micro/macroscale (phototropism, ${ }^{4}$ proton gradients that drive the beating of flagella, ${ }^{5}$ the motion of bacteria directed by chemotaxis $^{6}$ ), chemical signals correlated with a structural response, reconfiguration, or movement lie at the heart of many biological processes. Such diversity in the materials systems and mechanisms provides for an equally large range of elegant applications of chemomechanical actuators. ${ }^{7}$ The beating of cilia, hair-like cellular structures, in the respiratory tract helps to prevent biofilm formation, ${ }^{8}$ while the opening and closing of pedicellaria, flower-like extensions on the surfaces of sea urchins, are used for body cleaning and food capture. ${ }^{9}$ Cephalopods, such as octopus and squid, are artful masters of camouflage, making use of pigment-containing cells (chromatophores) that expand and contract to allow the animals to rapidly change color. ${ }^{10}$ The widespread demonstrated importance and versatility of such naturally occurring chemo-mechanical systems, particularly their extensive diversity in terms of scale, mechanism, function, and structure, serve as inspiration for the development of "smart" materials that are able to autonomously sense and adapt via self-regulated structural reconfiguration. Understanding and harnessing the power of combining chemically-induced mechanical responses with meaningful outcomes in terms of structure-function relationships, switchable surface properties, force generation, or directed chemical cascades, will be extremely valuable for designing responsive and adaptive systems. ${ }^{11-21}$ 
From among the many classes of stimuli-responsive materials, hydrogels in particular have received substantial attention due to their ability to translate a wide range of chemical signals into a mechanical output in the form of the gel's reversible volume change. Responsive hydrogels are crosslinked polymer networks which change volume in aqueous environments in response to stimuli including temperature, $\mathrm{pH}$, humidity, light, biomolecules, salt, magnetic and electric fields, redox state, etc. ${ }^{22}$ One popular use of this chemo-mechanical response of hydrogels is that of an artificial "muscle". For instance, when combined with "skeletal" arrays of high-aspect-ratio (HAR) structures, the hydrogel muscle is able to reversibly reconfigure/bend the embedded nano/microstructures, providing a means to switch surface properties and geometries in response to environmental cues..$^{23-30}$ The interplay between controllable parameters such as the location and type of stimulus, ${ }^{23,26,28}$ the surface chemistry, ${ }^{28}$ geometry, ${ }^{26}$ and mechanical properties of HAR structures, ${ }^{23,25}$ and the patterning and topography of the hydrogel within the confinement of the structure ${ }^{25,27}$ provide for a highly tunable and customizable chemo-mechanical hybrid system based on just a few individual material components. ${ }^{31}$ In this review, we explore these different modes of chemo-mechanical actuation, examining how various chemical inputs as well as the physical combination of gel and HAR structure lead to a diversity of responses, actuation patterns, and functions. We describe systems in which the gels are either surface attached ${ }^{23-26}$ or tethered to the HAR structures,${ }^{27}$ responding to homogenous chemical stimuli, ${ }^{23-28}$ gradient stimuli, ${ }^{29}$ and/or combinatorial stimuli, ${ }^{27}$ and with programmable actuation directions and bending angles. ${ }^{27,29}$ We explore how integration of the hybrid surfaces within different fluidic environments enables compartmentalized and spatially patterned application of stimuli further expanding the controllability of the chemo-mechanical response..$^{26,28}$ Finally, we examine how such hybrid surfaces can be used to mediate chemo-mechano-chemical processes including feedback loops, laying the foundation for unique routes to the dynamic control of mechanically-mediated chemical reactions. ${ }^{28,30}$

\section{Hybrid Systems: Modes of Actuation}

The hybrid systems we discuss here are all built from the same two fundamental components: 1) nano/microscale structures which provide the skeletal foundation for the hybrid surface and are to be dynamically reconfigured, and 2) a responsive hydrogel polymerized within the 
confinement of, and covalently attached to, the structures such that a volume-phase transition of the gel induces reconfiguration of the structures (Figure 1).

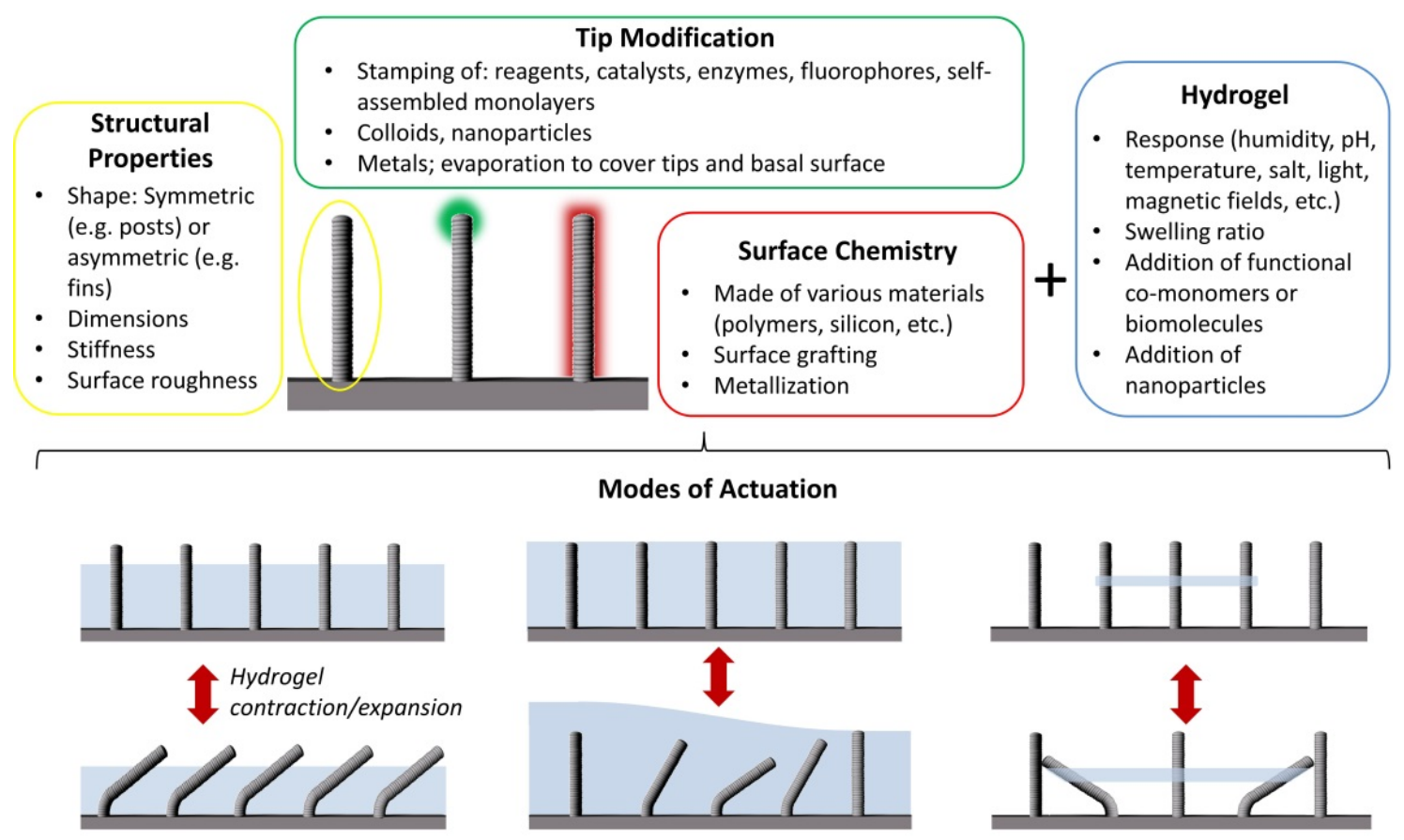

Figure 1. Hydrogel-actuated hybrid systems are highly customizable, both in their chemical and mechanical properties. In addition to the tunability of each material (embedded structures or hydrogel) there are also various ways to combine them such to create a range of modes of actuation each with different chemo-mechanical behavior. Bottom left to right: large-area homogeneous actuation, localized actuation within a well-defined region, and localized radial actuation from tethered hydrogel pads.

Both the skeletal structures and the hydrogel can be chemically and physically modified in many ways to create a diverse set of surfaces with tunable actuation and surface properties. The structures themselves can be made from a variety of materials such as silicon ${ }^{23}$ or numerous polymers and can be designed to have a range of geometries..$^{25,32}$ The structures' tips can be selectively modified, such as evaporation of metal ${ }^{33}$ or by stamping of chemicals, ${ }^{28,34}$ enzymes, ${ }^{30}$ or nanoparticles. ${ }^{35}$ The hydrogel used is similarly variable in its chemical composition depending on the desired stimulus sensitivity, swelling ratio, optical properties, etc. For all hybrid systems described herein this gel volume-phase transition, and therefore actuation, is highly reversible and the actuation can be cycled many times with little system fatigue. With just these two materials, varying their chemical identity or surface functionalization and how the 
hydrogel is physically integrated or configured within the structures leads to a variety of actuation mechanisms that can be selectively used to tailor the actuation response.

\subsection{Chemo-mechanical actuation of HAR structures in surface-attached hydrogel films}

In this mode of actuation the hydrogel is covalently attached to both the HAR structures and the basal surface (Figure 2a). With these constraints, the gel cannot expand/contract laterally as it changes volume, so its only option is to grow and shrink in thickness. ${ }^{36}$ However, the gel also may not slide up and down the structures; thus as it proceeds from the swollen state to the contracted state, its vertical shrinkage generates downward force on the embedded structures. Ultimately, this force generates instability in the vertically oriented structures and induces them to buckle, or tilt, in similar directions, allowing the gel to contract further and reach an equilibrium state. ${ }^{36}$ The structures thus actuate between two orientations upon exposure to a uniform, homogenous stimulus: 1) upright when the hydrogel is swollen and 2) bent when the hydrogel is contracted (Figure 2a). A variety of types of gel chemistries all exhibit this same mechanism. For example, with humidity-responsive hydrogel such as polyacrylamide (PAAm), structures are upright when the gel is hydrated and bent when the gel is dry. ${ }^{23-25}$ For temperatureresponsive poly( $N$-isopropylacrylamide) (PNIPAAm), the structures are upright when the temperature is below the lower critical solution temperature (LCST) of $32^{\circ} \mathrm{C}$ and bent when the temperature is raised above the LCST. ${ }^{28}$ For a $\mathrm{pH}$-responsive hydrogel, poly(acrylic acid-coacrylamide) (poly(AAc-co-AAm)), the hydrogel transitions between a contracted state where structures are bent and a swollen state where structures are upright between $\mathrm{pH}=4-5$ in accordance with the $\mathrm{pK}_{\mathrm{a}}$ of the acrylic acid. ${ }^{26}$

The structures generally bend in reproducible directions with each actuation cycle. However, the particular bending direction is influenced by the HAR geometry. For example, symmetrical HAR post structures, which have no geometrically preferred bending direction, are observed to bend in small directional domains that diverge across a surface and are sensitive to localized asymmetries in the hydrogel or surface defects (Figure 2b). In contrast, asymmetric fin structures possessing a preferred bending orientation in accordance with their geometry generate large areas $\left(\mathrm{cm}^{2}\right.$ at least) of highly uniform directional actuation (Figure 2c). ${ }^{26}$ These surfaces can be used to modulate transparency or reflectivity in response to changes in the external environment, such as humidity or temperature, which could be useful for "smart" windows; if the 
fin structures are coated on one side by shadow evaporation of metal, then when the fins stand upright the surface is highly transparent, but when the gel contracts and the fins bend over, the surface becomes reflective and opaque. ${ }^{33}$

The stiffness of the structures relative to the force that can be exerted by the gel dictates whether or not actuation can take place. If the structures are made from a stiff material such as silicon (Young's modulus $E \approx 200 \mathrm{GPa}$ ), very high aspect ratios of the structures (low effective stiffness) must be used to provide responsiveness. ${ }^{23}$ To induce actuation of lower aspect ratio microscale structures, soft flexible polymeric materials such as epoxies, polyurethanes, polydimethylsiloxane (PDMS), etc. that have stiffness in the MPa to low GPa range can be used. $^{25}$ The exception is the case in which structures are free-standing in the gel and tilt rather than bend upon gel contraction, therefore removing any stiffness requirements. ${ }^{23}$
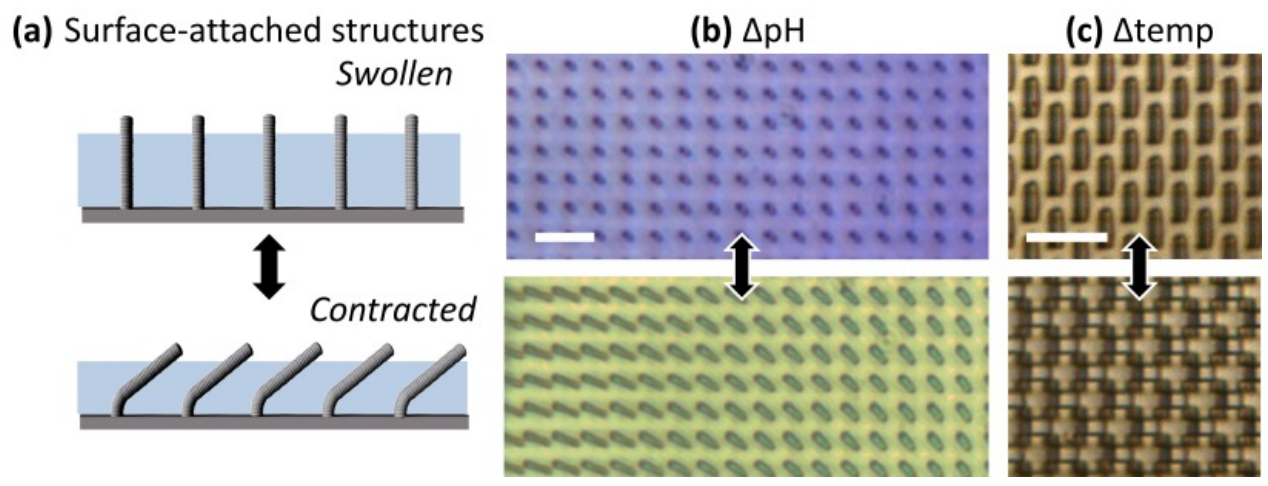

Figure 2. (a) HAR structures reversibly bend upon contraction/expansion of hydrogel. (b) Optical micrographs of micropost actuation with a poly(AAc-co-AAm) hydrogel (color from $\mathrm{pH}$ indicator) showing how the bending direction begins to diverge over short distances. (c) Microfin actuation with a PNIPAAm hydrogel that displays uniform actuation. Scale $=20 \mu \mathrm{m}$. Adapted from Reference 26 and 28 .

\subsection{Hybrid actuation systems responsive to chemical gradients}

While surfaces described in Section 2.1 allow for generation of actuation over large areas by application of homogeneous stimulus, it is sometimes desirable to define localized regions of actuation with tunable bending directions. This complementary mode of actuation utilizes surfaces of composition similar to those described in Section 2.1 (hydrogel films covalently attached to the HAR structures and basal surface) but actuation is instead accomplished through the use of chemical gradients. ${ }^{29}$ Structures remain standing upright both when the gel is expanded and contracted, showing no actuation in the presence of a homogeneous stimulus 
despite responsive volume changes in the gel. Instead, the structures bend specifically in response to spatial changes, or gradients, in the stimulus. This selectivity is achieved by, for example, polymerizing the hydrogel under conditions when the structures start out upright when the gel is contracted such that cycles of homogenous, vertical gel expansion and contraction exert no downward force upon the embedded structures. Bending is thus driven specifically by local regions of lateral gel expansion and contraction determined by a stimulus gradient (Figure $3 a){ }^{29}$

(a)
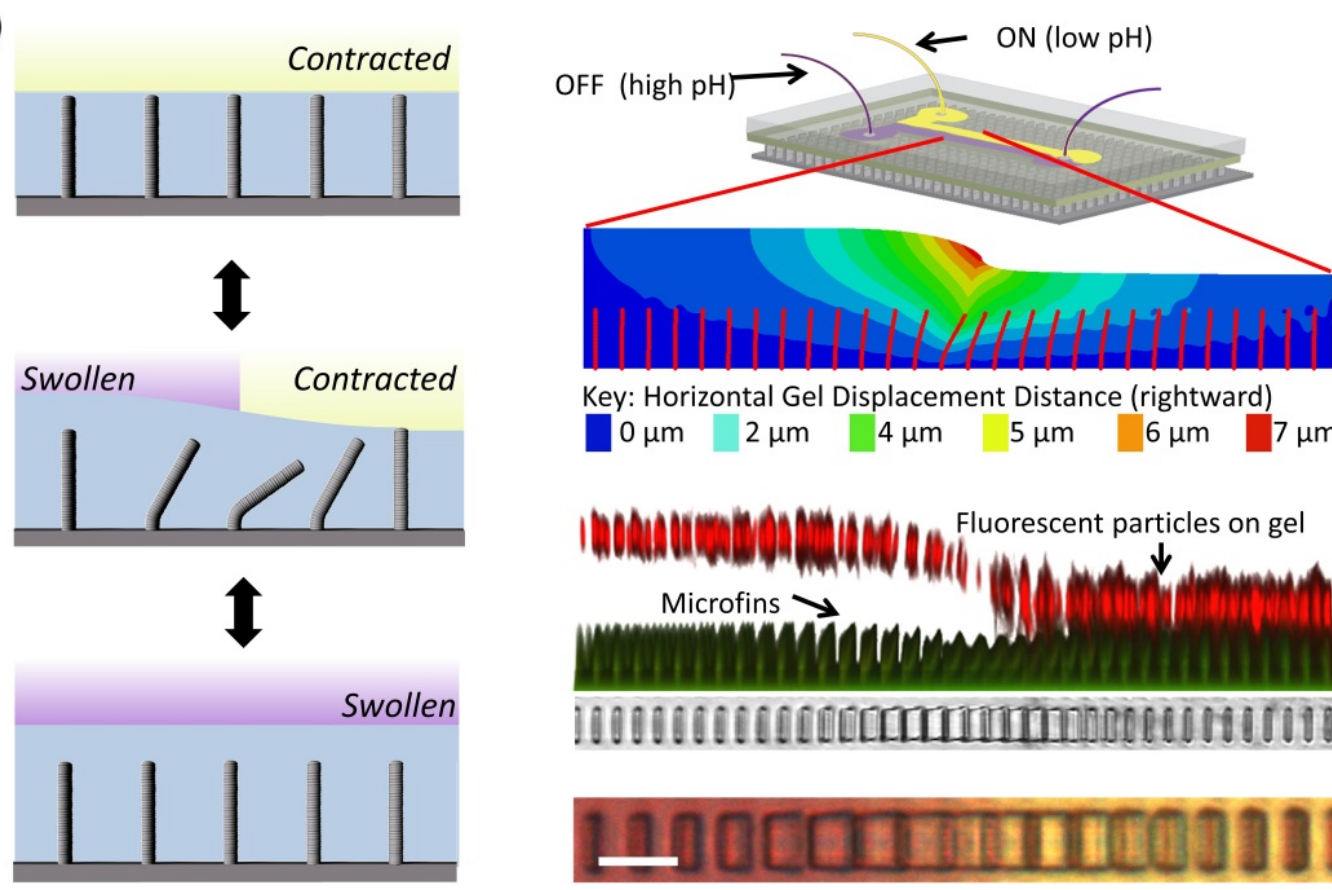

(b)

(c)
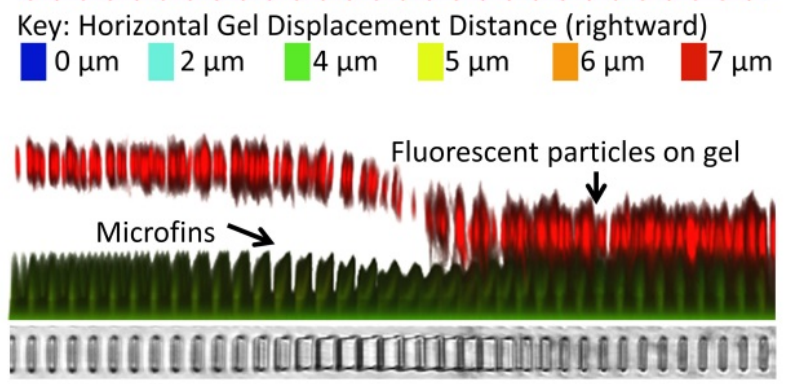

(d)

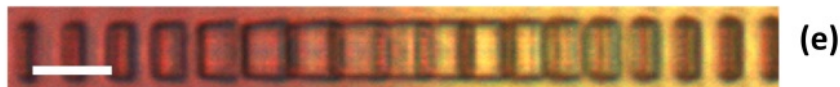

Figure 3. Actuation induced by the presence of a chemical gradient. (a) Schematic demonstrating the mechanism. (b) Diagram of the microfluidic setup used to impose a $\mathrm{pH}$ gradient upon a hybrid surface. (c) Modeling showing that the hydrogel at the gradient center shifts laterally, toward the contracted gel, driving the actuation. (d) Confocal Z-stack cross-section showing the topography of the hydrogel under the influence of a pH gradient. An optical micrograph of the same area is aligned underneath. (e) Optical micrograph allowing visualization of the $\mathrm{pH}$ gradient and showing actuation of structures toward the contracted gel (acid). Color arises from bromophenol blue $\mathrm{pH}$ indicator. Scale $=10 \mu \mathrm{m}$. Adapted from Reference 29.

Modeling and simulation, as well as the use of confocal microscopy to visualize the surface topography of the gel, elucidate this actuation mechanism. When a stimulus gradient is present across the surface, such as a $\mathrm{pH}$ gradient in the case of the poly(AAc-co-AAm) hybrid surfaces (Figure $3 b$ ), the gel is locally displaced laterally, in addition to vertically, across this gradient 
with the swollen part of the gel expanding toward the contracted gel (Figure 3c). This is in contrast to homogenous gel expansion and contraction where the gel only swells vertically. This lateral shifting, which always occurs in the direction toward the contracted gel region, is the driving force behind the actuation. The structures therefore always bend toward the contracted gel, and only within the transition zone where the gel profile is sloped, as visualized by confocal microscopy (Figure 3d). Farther from the gradient center, where the gel has reached an equilibrium swollen or contracted state, there is no net force on the structures and they remain upright. The width of the region over which structures are bent correlates with the gel thickness, which is the only relevant length scale in the system - thicker gels require a wider region over which to reach the equilibrium contracted and swollen states, so the area over which the gel laterally shifts also increases accordingly, allowing for tunable actuation areas from tens to hundreds of microns across the $\mathrm{pH}$ gradient.

Since the actuation direction is reversibly controllable with the shape, location, and direction of the applied chemical gradient, this mechanism provides tunability of the structural reconfiguration not possible with other mechanisms. Additionally, the surface acts as a sensor, operating as a visual vector gradient and reporting not only local variations in the chemical environment, but also any changes in topography of the hydrogel that might arise from other phenomena, such as gel creasing. ${ }^{37}$ Furthermore, such surfaces retain their ability to actuate in response to humidity such that upon drying all structures bend via the mechanism described in Section 2.1. This added ability to chemically manipulate the actuation and induce either localized or bulk actuation in the same system by switching from submerged to dry conditions can significantly enhance versatility and controllability of the responsive hybrid surface, rendering it capable of countless other response scenarios.

\subsection{Thin hydrogel pads tethered to HAR structures}

If the hydrogel is attached only to the structures and not to the basal surface, as opposed to the systems discussed in Sections 2.1-2.2, the gel is far less constrained within the surface plane, generating yet another distinct chemo-mechanical responsive actuation mode. ${ }^{27}$ The driving force behind actuation in this case is lateral gel expansion and contraction parallel to the surface plane induced by changes in a homogenous stimulus (Figure 4a,b). Structures are actuated radially inward and outward, with those near the edges of the gel receiving the greatest amount 
of force and bending the most and those near the middle receiving less net force and remaining relatively undisturbed. Since the gel experiences significantly lower constraint in the lateral dimension due to the decreased surface confinement, the gels exhibit large in-plane swelling ratios, providing a route to tuning the structures' bending angles across wide ranges with small changes in applied stimuli (Figure 4c). Because the gel expansion is radially outward, the bending direction is simply determined by the overall shape of the gel. Therefore, it is possible to achieve a specific actuation pattern by rationally designing the geometry and placement of the gels on the surface in combination with a corresponding stimulus.

(a)

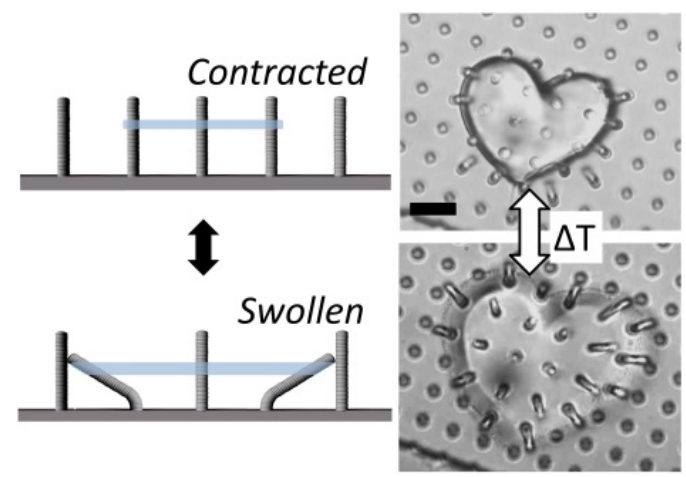

(b)

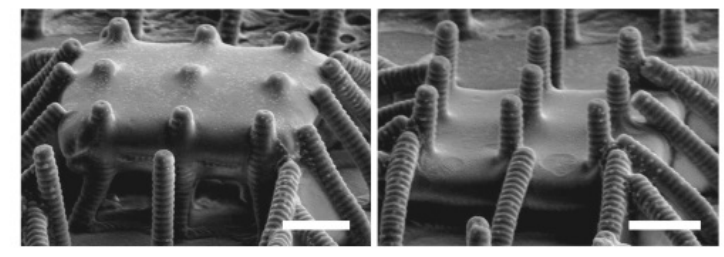

(c)

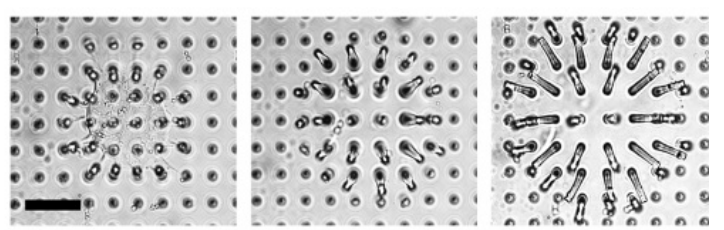

$\mathrm{pH}=2 \quad \mathrm{pH}=4.5 \quad \mathrm{pH}=8.8$

(d)

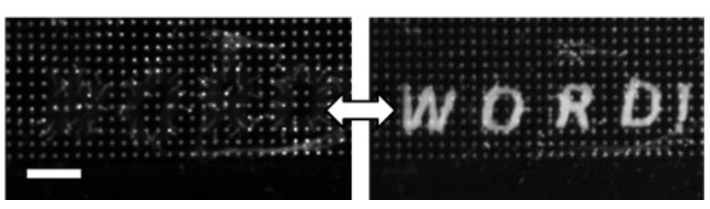

Figure 4. Actuation induced by volume change of thin hydrogel pads. (a) Schematic of the actuation mechanism. Right: optical micrographs showing PNIPAAm hydrogel-actuated structures. Scale $=10 \mu \mathrm{m}$. (b) Scanning electron micrographs showing thin pads of hydrogel near the top and bottom of posts, demonstrating precise 3D control over placement. Scale $=5 \mu \mathrm{m}$. (c) Optical images of a circular $\mathrm{pH}-$ responsive hydrogel at three different $\mathrm{pH}$ values demonstrating tunability of the post bending angles. While posts near the center of the gel show little change in bending angle, posts near the edge can be almost entirely bent over to $70^{\circ}$. (d) Rhodamine covalently incorporated into poly(AAc-co-AAm) 
hydrogel changes concentration as the gel significantly swells and shrinks thereby reversibly revealing and erasing the message "Word!". Scale $=40 \mu \mathrm{m}$. Adapted from Reference 27 .

Generating thin hydrogels that are not surface attached, yet are still integrated with the HAR structures, is achieved by using multiphoton lithography (MPL). ${ }^{27}$ A hydrogel pad as thin as 2 $\mu \mathrm{m}$ can be integrated anywhere along the lengths of the HAR structures, allowing the gel to be free from attachment to the basal surface, but not free-floating (Figure $4 b$ ). Since the gels are far more free from constraints they exhibit large swelling ratios, as can easily be visualized by the appearance and disappearance of a message drawn in rhodamine-modified poly(AAc-co-AAm) (Figure 4d). When the gel is contracted the dye is concentrated and the message is visible, but when the gel swells the dye is so significantly diluted that the signal is no longer seen under the same conditions. ${ }^{27}$ Such optical response is reminiscent of chromatophores in cephalopods, where the expansion/contraction of muscles switch on/off the appearance of color. ${ }^{10}$

\section{Integration of Hybrid Surfaces with Fluidic Systems}

With the exception of humidity-responsive gels for which the stimulus is the presence or absence of water or vapor, most responsive hydrogels require a fluidic environment to apply a stimulus. Consequently, development of different approaches for integrating hybrid surfaces within fluidic/microfluidic environments is essential to providing variable routes for patterning and compartmentalizing application of stimuli. A number of different configurations of the hybrid surface and its components (hydrogel and structures) with respect to the environment collectively allow for both horizontal and vertical compartmentalization of the stimulus to enable enhanced control over location, pattern, and rate of stimulus application.

The simplest fluidic environment is a bulk solution that can be changed externally, for example, by adding acid/base to induce volume change in a $\mathrm{pH}$-responsive gel or by external heating to induce volume change in a temperature-responsive gel. Since the solution is homogenous, actuation is induced simultaneously over the whole sample (Figure 5a). For more localized actuation, the hybrid surfaces can be integrated within microfluidic systems (Figure $5 b, c)$ with the actuating surface serving as the bottom of the microfluidic channel, laser-cut double sided adhesives defining the walls of the channel, and PDMS as the channel top. ${ }^{26}$ 
(a)

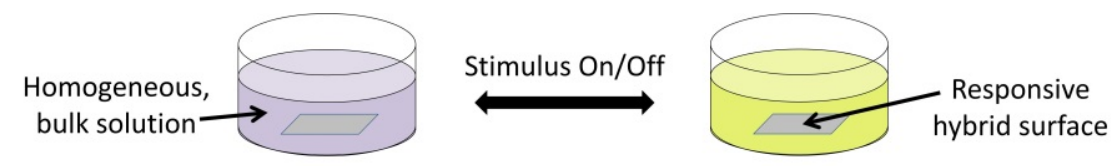

(b)

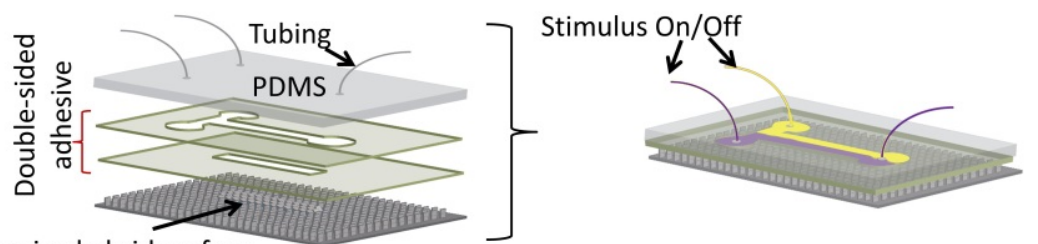

Responsive hybrid surface

(c)

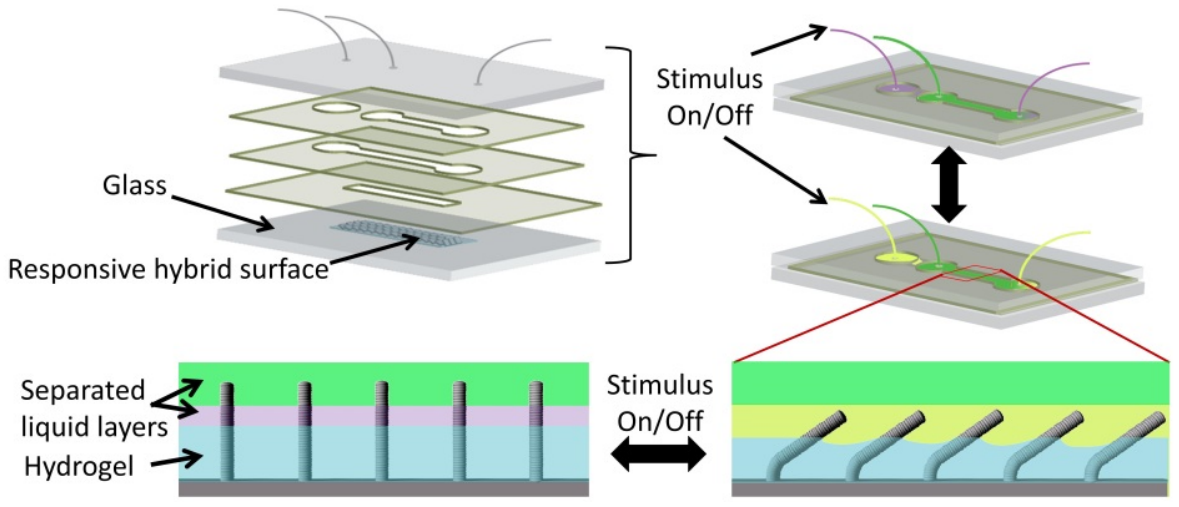

Figure 5. Schematics showing (a) application of a homogenous stimulus to a hybrid surface, (b) integration of a hybrid surface into a microfluidic channel designed to apply horizontal laminar flow of different stimuli, and (c) integration of a hybrid surface into a microfluidic channel designed to apply vertical laminar flow of two liquids on top of each other. Cross-section of the system in (c) shows that the tips of the structures and the gel can be separated into different environments and structures may be moved in and out of the upper liquid environment via actuation.

Integration within microfluidic systems provides the ability to compartmentalize stimuli across the surface, both horizontally (Figure 5b) and vertically (Figure 5c). Horizontal compartmentalization, in which two solutions flow side by side, creates a well-defined chemical gradient that can drive actuation of structures as described in Section 2.2. ${ }^{26,29}$ Vertical compartmentalization (two solutions layered on top of each other) allows the chemical environment of the hydrogel to be distinct from, and independent of, the environment of the structures tips that protrude from the gel. ${ }^{28,30}$ Vertical compartmentalization can be achieved without microfluidics if the two solutions are immiscible and create a stable bilayer, but for entirely aqueous systems laminar flow can be used. The vertical compartmentalization can be designed so that the interface of the two solutions falls just below the tips of the structures, such that upon actuation the structure tips move in and out of the upper liquid layer (Figure 5c 
bottom). ${ }^{28}$ This vertical configuration is especially interesting as it potentially allows for actuating structures to serve as a transport and sorting mechanism, if the structure tips are functionalized to bind target molecules or even cells in the upper layer and release them within the lower collection layer.

\section{Combinatorial Approaches: Structural and Chemical Manipulation of Actuation using Complex, Patterned Asymmetric Forces}

The discussed actuation mechanisms provide a set of general routes for the predictable actuation of arrays of HAR structures via the mechanical forces exerted by responsive hydrogels as they expand/contract. These modes of actuation are complementary and can act cooperatively such that combinations of multiple approaches allow for even more complex actuation scenarios than a single mode alone. Through this combination of mechanisms, as well as by localized control over chemical stimuli and gel and HAR structure geometries, we have uncovered new ways to generate asymmetric forces on the embedded HAR structures, inducing them to bend in programmable directions.

For example, if we introduce variations into the hydrogel thickness and topography rather than using a uniform hydrogel film we can generate pattered actuation directions of the HAR structures. $^{23,25}$ Such gel surface topography can be controlled by polymerizing the hydrogel under the influence of either a photomask or a 3-D topographically patterned confining surface (Figure 6a). Since a thicker hydrogel has a greater propensity for absolute volume contraction, it acts as a stronger "muscle" and embedded HAR structures are pushed or pulled by the thicker gel. ${ }^{25}$ For example, the "flowering" actuation of microposts embedded in a $\mathrm{pH}$-responsive gel shown in Figure $6 \mathrm{~b}$ can be created using a photomask with an array of squares. We further observe that three different states are accessible; posts are bent outward when the gel is fully swollen in basic conditions; posts primarily stand upright when the gel is contracted at $\mathrm{pH}<4$, and the posts are bent inward toward the center of the gel square when the gel is fully contracted and dry. The actuation is highly reproducible and structures repeatedly bend in the same preprogrammed direction as determined by the gel topography with every actuation cycle. This patterned movement of the embedded structures can be modeled as a function of hydrogel thickness/topography $;{ }^{25}$ Figure $6 \mathrm{c}$ shows a model implemented in the finite element package, 
ABAQUS, to describe the movement and strain in the system as a function of hydrogel swelling and contraction for the simple case of humidity-responsive hydrogel.

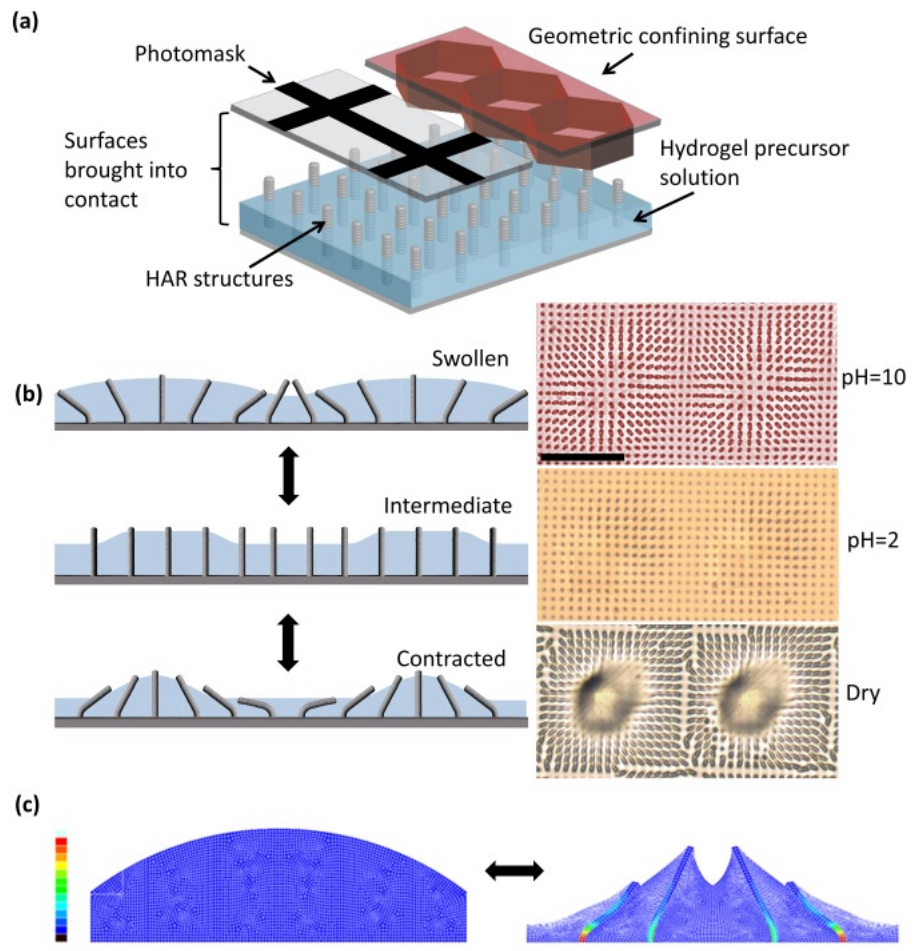

(d)

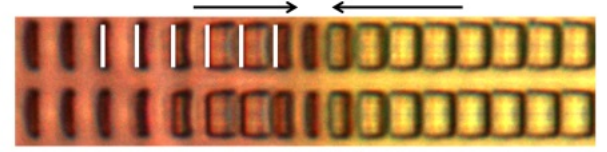

(e)

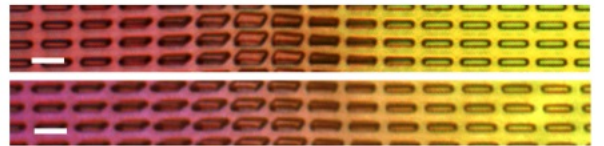

(f)

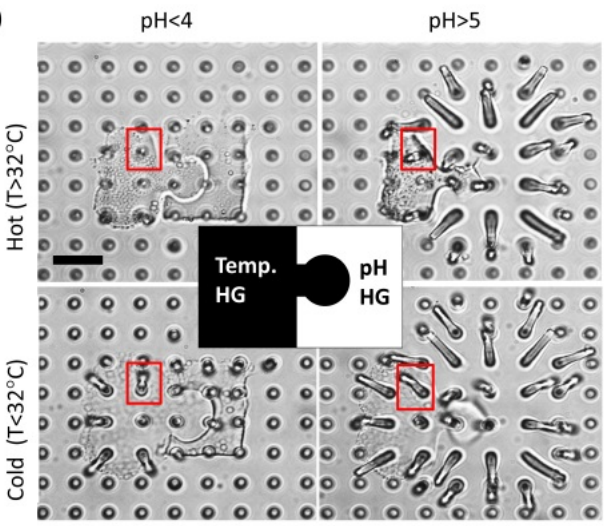

Figure 6. Approaches to patterned actuation. (a) Schematic of fabrication techniques; a photomask or 3Dpatterned geometric confining surface can be used to control hydrogel topography. (b) Schematic of actuation mechanism for hydrogel film with patterned topography. Right: optical micrographs of a $\mathrm{pH}-$ responsive gel in the fully swollen state (base), intermediate state (acid) and contracted state (dry) in correlation with the schematic. Scale $=100 \mu \mathrm{m}$. (c) 2D finite element simulation showing bending of polymer posts embedded in a responsive hydrogel layer with varied thickness. As the hydrogel swells/contracts the structures are strained, indicated by the color scale (red is large strain). (d) A hybrid surface that responds to bulk stimulus under the influence of a $\mathrm{pH}$-gradient exhibits backward-bending behavior over short distances. White bars mark the base of the structures to help visualize bending directions which are marked with arrows. (e) Application of the $\mathrm{pH}$-gradient at different angles relative to the length of the anisotropic fin results in tunable bending angles. Scale $=10 \mu \mathrm{m}$. (f) Temperature and $\mathrm{pH}$-responsive hydrogels patterned in sequence with precise registration to form interlocking puzzle piece shapes. An exemplary post sitting at the interface between the two gels (red box) feels the effects of both "muscles" and bends in four different directions according to combinatorial stimuli. Scale $=10 \mu \mathrm{m}$. Adapted from References 25, 26, 27, and 29.

Further combining both structural/topographical and chemical approaches leads to an even greater diversity of actuation possibilities. For example, application of a chemical stimulus 
gradient on a hybrid surface which does actuate in response to homogenous bulk stimulus can effectively induce specific structures to reverse bending directions over short distances (Figure 6d). Applying a chemical gradient along different axes of an asymmetric microfins also results in a tunable range of bending orientations (Figure 6e). ${ }^{29}$

It is also possible to generate localized asymmetries in gel forces and control bending direction of individual structures through the combination of chemical signals by using multiple types of responsive hydrogel (separately controlled "muscles") to manipulate a single HAR structure. Using MPL, which provides the ability to pattern different hydrogels in sequence with precise

registration, two (or more) gels can be positioned on either side of a HAR structure. ${ }^{27}$ Structures at this interface between the gels are pushed or pulled by the expansion and contraction of both gels simultaneously such that the bending direction is sensitive to different combinations of chemical signals, leading to multiple situation-specific bending orientations (Figure 6f).

\section{Manipulation of the Chemical Environment using Hybrid Actuation Systems}

Hydrogel-driven hybrid actuation systems are keen examples of chemo (C)-mechanical (M) actuators $(\mathrm{C} \rightarrow \mathrm{M})$; the hydrogel provides the chemical sensitivity and the embedded HAR structures provide the basis for translation into directed motion. Taking this a step further, we can use the mechanical actuation of the hybrid system to induce a programmed chemical output $\left(\mathrm{C}_{2}\right)$ distinct from the chemistry inside the hydrogel $\left(\mathrm{C}_{1}\right)$ in a chemo-mechano-chemical cycle $\left(\mathrm{C}_{1} \rightarrow \mathrm{M} \rightarrow \mathrm{C}_{2}\right)$, opening routes for unique mechanistic control over dynamic chemical reactions, generation of output products including biochemicals, light, heat, or gas, and even the possibility of feedback loops if there is correlation between the chemical output and the hydrogel sensitivity.

To synchronize the responsive actuation such that the output reaction can be turned on and off by movement of the HAR structures, the chemical change driving the actuation $\left(\mathrm{C}_{1} \rightarrow \mathrm{M}\right)$ must be physically separated from the reaction that produces the chemical output $\left(M \rightarrow C_{2}\right)$. This is achieved by vertical compartmentalization systems discussed in Section 3: either by microfluidic layering, with two miscible liquids flowing on top of one another (Figure 5c) or with a static interface between two immiscible liquids. In this way, the chemical stimulus $\mathrm{C}_{1}$, which induces the hydrogel to expand/contract and drives actuation, can be introduced to the bottom layer, 
while the top layer contains reagents that will be acted upon by a catalyst attached to the structure tips $\left(\mathrm{C}_{2}\right)$. When structures stand upright, their tips enter the reagent environment and the reaction is turned "on"; when the structures bend, their tips exit the reagent layer and the reaction is turned "off". Because the chemical environments are physically separated, the system allows for the coupling of a wide variety of chemical inputs and outputs which may otherwise be chemically incompatible. For example, the use of $\mathrm{pH}$ as the chemical stimulus $\left(\mathrm{C}_{1}\right)$ can be used to turn on/off the fluorescence of a dye, the generation of oxygen from Pt-catalyzed decomposition of hydrogen peroxide, or the activity of a biochemical reaction such as luciferasecatalyzed oxidation of luciferin. ${ }^{28,30}$

If the chemical output itself triggers a differential response in the hydrogel and subsequently induces actuation, then it is possible to create an autonomous feedback loop within the hybrid system. ${ }^{28}$ For example, a temperature-responsive PNIPAAm hydrogel can be paired with a variety of exothermic output reactions to generate a tunable homeostatic feedback loop that autonomously maintains the surface temperature at a programmed set-point (Figure 7a). When the temperature is below the LCST of PNIPAAm $\left(\mathrm{T}<32^{\circ} \mathrm{C}\right)$, the gel is swollen and the structures stand upright thereby turning on an exothermic reaction. As the solution heats to above the LCST, the hydrogel contracts causing the structures to bend, turning off the reaction to allow a cooling phase. When the solution cools to below the LCST, the cycle starts again, regulating the oscillating temperature within a few degrees of the LCST indefinitely, as long as the reagents are replenished.

Such feedback systems are tunable based on parameters such as the responsiveness of the hydrogel, exothermic reaction used, rate of heat production, structure geometry, and the location of the bilayer liquid interface. If the LCST is altered by changing the hydrophobicity of the hydrogel, then the temperature range over which the surface fluctuates changes accordingly (Figure 7b); a system where the gel has a LCST $=32^{\circ} \mathrm{C}$ maintains temperatures of $31.0-33.8^{\circ} \mathrm{C}$ but a system incorporating a gel with $\mathrm{LCST}=27.8^{\circ} \mathrm{C}$ maintains temperatures of $27.1-29.7^{\circ} \mathrm{C}$. Exothermic reactions demonstrated include the hydrosilylation of 1-hexene with triethylsilane, or 1-hexene with diphenylsilane, catalyzed $\mathrm{byH}_{2} \mathrm{PtCl}_{6}$, the decomposition of cumene hydroperoxide catalyzed by $\mathrm{Ph}_{3} \mathrm{CPF}_{6}$, and a click reaction between octylazide and phenylacetylene catalyzed by $\mathrm{Cu}\left(\mathrm{PPh}_{3}\right)_{2} \mathrm{NO}_{3}$. Each has its own characteristic kinetics and heat generation profiles that ultimately affect the dynamics of the feedback process. If the heating rate is higher, for instance, 
the amplitude of the temperature fluctuations increases and the oscillation period is shortened. Raising the bilayer interface results in smaller temperature fluctuations around a slightly lower temperature, presumably because the catalyst-bearing tips spend less time immersed in the reagent layer. Such geometric and chemical parameters lend the system a high degree of customizability.

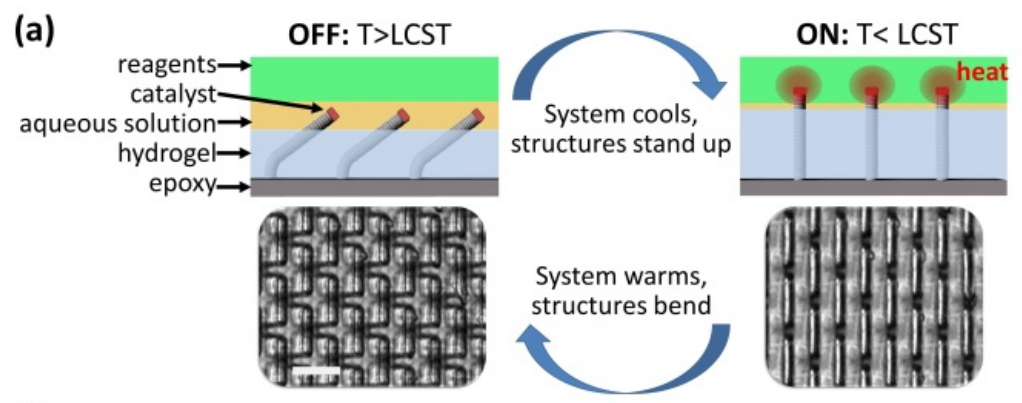

(b)

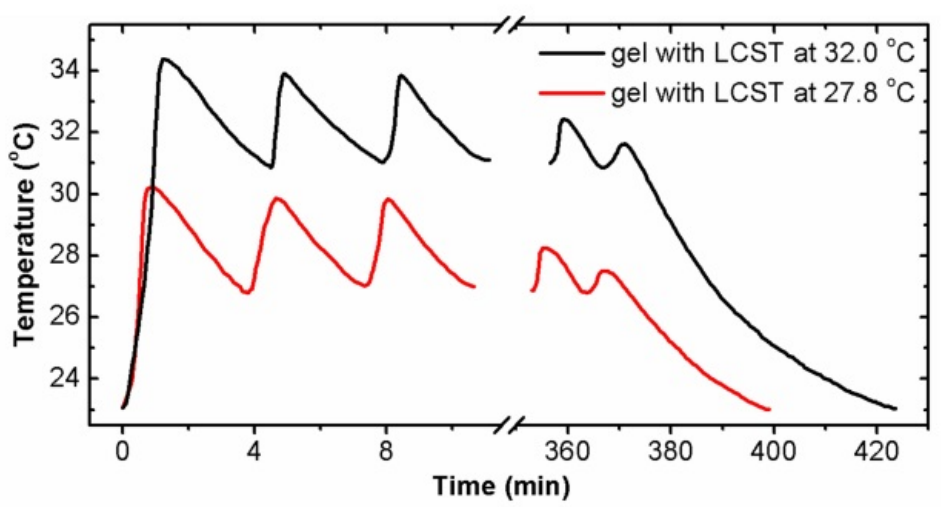

Figure 7. Chemo-mechanical feedback loops generated by actuation of a hybrid surface. (a) Schematic demonstrating the feedback mechanism of a temperature-regulating hybrid surface. Structure tips are coated with a catalyst that, upon entering the reagent layer, induces an exothermic reaction. This heat raises the surface temperature to above the LCST of the PNIPAAm, causing the gel to contract and the structures to bend, removing the catalyst from the reagent layer and turning off the reaction. The surface cools to below the LCST and the structures stand up, restarting the feedback cycle. Scale $=10 \mu \mathrm{m}$. (b) Surface temperature measurements for surfaces with two PNIPAAm-based hydrogels with different LCSTs. Adapted from Reference 28.

\section{Conclusions and Outlook}

Hybrid architectures, which take advantage of the unique properties generated by the interplay between multiple materials integrated into a coherent functional system, allow for the development of tunable chemo-mechanical responses otherwise unachievable by a single 
material alone. Indeed, by exploring various approaches to the combination of just two simple and well-characterized materials - an array of structures and a responsive hydrogel, - a far more complex set of chemo-mechanical actuation mechanisms has emerged. The hydrogel, which has a reversibly responsive volume change, acts as the "muscle" that provides force to reconfigure flexible HAR structures. By varying the HAR structure geometry and surface chemistry, the hydrogel chemistry, topography, location, and pattern, as well as the spatial presentation and combination of stimuli, we access a few fundamental mechanisms identified herein, which provide routes to higher complexity of chemo-mechanical actuation. Such modes of actuation offer means of achieving large-area homogenous actuation, highly localized actuation, patterned actuation, and variable directions of actuation. Taking these systems a step further, we have developed an approach to not only translate a chemical signal into the mechanical movement of microstructures but to then translate that mechanical movement into a coupled chemical output. Therefore, such actuators switch system/surface properties not only through chemo-mechanical means, such as using the structures' reconfiguration to alter adhesion, wetting behavior, or optical properties, but also through mechano-chemical means, creating outputs of light, heat, biomolecules, gas, etc., significantly extending the hybrid system's versatility. While we have shown examples that utilize temperature-, humidity- and $\mathrm{pH}$-responsive gels, additional systems are also being explored, such as those that actuate in response to light, pressure, highly localized actuation induced by laser heating of nanoparticle-embedded gels, and hybrid surfaces capable of microfluidic sorting of biomolecules or even cells.

Such materials that sense changes in their environment and functionally respond via chemomechano-chemical processes provide the foundation for next-generation self-regulating, selfsustaining, and autonomously adaptive "smart" systems. While fundamentally critical to the adaptive mechanisms and robustness of living systems, the ability to interchange mechanical and chemical inputs and outputs has proven difficult to replicate in artificial materials. While biology makes use of multi-step cascades, complex feedback processes, and ultra-sensitive chemical and mechanical receptors, we are still in the early stages of developing materials that exhibit comparatively simple chemo-mechanical responses. However, the great promise of such adaptive systems, applied to make self-camouflaging materials, actuating surfaces that prevent biofouling, or buildings that save energy by self-regulating temperature or light, continues to drive research in the field of smart, chemo-mechanical materials. 


\section{ACKNOWLEDGMENT}

We thank Dr. Alison Grinthal and Dr. Ximin He for their helpful discussions and comments during the preparation of this manuscript. The work was supported by the DOE under award DE-SC0005247 and by NSF under award CMMI-1124839.

\section{REFERENCES}

1. Cohen Stuart, M. A.; Huck, W. T. S.; Genzer, J.; Müller, M.; Ober, C.; Stamm, M.; Sukhorukov, G. B.; Szleifer, I.; Tsukruk, V. V.; Urban, M.; Winnik, F.; Zauscher, S.; Luzinov, I.; Minko, S. Emerging Applications of Stimuli-Responsive Polymer Materials. Nat. Mater. 2010, 9, 101-113.

2. Sambongi, Y.; Iko, Y.; Tanabe, M.; Omote, H.; Iwamoto-Kihara, A.; Ueda, I.; Yanagida, T.; Wada, Y.; Futai, M. Mechanical Rotation of the C Subunit Oligomer in ATP Synthase (F0F1): Direct Observation. Science 1999, 286, 1722-1724.

3. Monod, J.; Changeux, J.-P.; Jacob, F. Allosteric Proteins and Cellular Control Systems. J. Mol. Biol. 1963, 6, 306-329.

4. Christie, J. M.; Reymond, P.; Powell, G. K.; Bernasconi, P.; Raibekas, A. A.; Liscum, E.; Briggs, W. R. Arabidopsis NPH1: a Flavoprotein with the Properties of a Photoreceptor for Phototropism. Science 1998, 282, 1698-1701.

5. Manson, M. D.; Tedesco, P.; Berg, H. C.; Harold, F. M.; Van Der Drift, C. A Protonmotive Force Drives Bacterial Flagella. Proc. Natl. Acad. Sci. U.S.A. 1977, 74, 3060.

6. Alon, U.; Surette, M. G.; Barkai, N.; Leibler, S. Robustness in Bacterial Chemotaxis. Nature 1999, 397, 168-171.

7. Fratzl, P.; Barth, F. G. Biomaterial Systems for Mechanosensing and Actuation. Nature 2009, 462, 442-448.

8. Satir, P. The Physiology of Cilia and Mucociliary Interactions. Annu. Rev. Physiol. 1990, 52, 137-155.

9. Ghyoot, M.; Ridder, C. D.; Jangoux, M. Fine Structure and Presumed Functions of the Pedicellariae of Echinocardium cordatum (Echinodermata, Echinoida). Zoomorphology 1987, 106, 279-288.

10. Sutherland, R. L.; Mäthger, L. M.; Hanlon, R. T.; Urbas, A. M.; Stone, M. O. Cephalopod Coloration Model. I. Squid Chromatophores and Iridophores. J. Opt. Soc. Am. 2008, 25, 588-599.

11. Stupp, S. I.; Braun, P. V. Molecular Manipulation of Microstructures: Biomaterials, Ceramics, and Semiconductors. Science 1997, 277, 1242-1248.

12. Lahann, J.; Langer, R. Smart Materials with Dynamically Controllable Surfaces. MRS Bulletin 2005, 30, 185-188.

13. Koumura, N.; Zijlstra, R. W.; van Delden, R. A.; Harada, N.; Feringa, B. L. Light-Driven Monodirectional Molecular Rotor. Nature 1999, 401, 152-155.

14. Paxton, W. F.; Sundararajan, S.; Mallouk, T. E.; Sen, A. Chemical Locomotion. Angew. Chem. Int. Ed. 2006, 45, 5420-5429. 
15. Krieg, E.; Shirman, E.; Weissman, H.; Shimoni, E.; Wolf, S. G.; Pinkas, I.; Rybtchinski, B. Supramolecular Gel based on a Perylene Diimide Dye: Multiple Stimuli Responsiveness, Robustness, and Photofunction. J. Am. Chem. Soc. 2009, 131, 14365-14373.

16. Luzinov, I.; Minko, S.; Tsukruk, V. V. Adaptive and Responsive Ssurfaces Through Controlled Reorganization of Interfacial Polymer Layers. Prog. Polym. Sci. 2004, 29, 635-698.

17. Palmer, L. C.; Velichko, Y. S.; de la Cruz, M. O.; Stupp, S. I. Supramolecular Self-Assembly Codes for Functional Structures. Philos. Trans. R. Soc. London, Ser. A. 2007, 365, 1417-1433.

18. Reddy, S.; Arzt, E.; del Campo, A. Bioinspired Surfaces with Switchable Adhesion. Adv. Mater. 2007, 19, 3833-3837.

19. Bosman, A. W.; Sijbesma, R. P.; Meijer, E. Supramolecular Polymers at Work. Mater. Today 2004, 7, 34-39.

20. Saha, S.; Copic, D.; Bhaskar, S.; Clay, N.; Donini, A.; Hart, A. J.; Lahann, J. Chemically Controlled Bending of Compositionally Anisotropic Microcylinders. Angew. Chem. Int. Ed. 2012, 51, 660-665.

21. Grinthal, A.; Aizenberg, J. Adaptive All the Way Down: Building Responsive Materials from Hierarchies of Chemomechanical Feedback. Chem. Soc. Rev. 2013, 42, 7072-7085.

22. Gupta, P.; Vermani, K.; Garg, S. Hydrogels: from Controlled Release to pH-Responsive Drug Delivery. Drug Discovery Today 2002, 7, 569-579.

23. Sidorenko, A.; Krupenkin, T.; Taylor, A.; Fratzl, P.; Aizenberg, J. Reversible Switching of Hydrogel-Actuated Nanostructures into Complex Micropatterns. Science 2007, 315, 487-490.

24. Sidorenko, A.; Krupenkin, T.; Aizenberg, J. Controlled Switching of the Wetting Behavior of Biomimetic Surfaces with Hydrogel-Supported Nanostructures. J. Mater. Chem. 2008, 18, 38413846.

25. Kim, P.; Zarzar, L. D.; Zhao, X.; Sidorenko, A.; Aizenberg, J. Microbristle in Gels: toward AllPolymer Reconfigurable Hybrid Surfaces. Soft Matter 2010, 6, 750-755.

26. Zarzar, L. D.; Kim, P.; Aizenberg, J. Bio-inspired Design of Submerged Hydrogel-Actuated Polymer Microstructures Operating in Response to pH. Adv. Mater. 2011, 23, 1442-1446.

27. Zarzar, L. D.; Kim, P.; Kolle, M.; Brinker, C. J.; Aizenberg, J.; Kaehr, B. Direct Writing and Actuation of 3D-Patterned Hydrogel Pads on Micropillar Supports. Angew. Chem. Int. Ed. 2011, 50, 9356-9360.

28. He, X.; Aizenberg, M.; Kuksenok, O.; Zarzar, L. D.; Shastri, A.; Balazs, A. C.; Aizenberg, J. Synthetic Homeostatic Materials with Chemo-mechano-chemical Self-Regulation. Nature 2012, 487, 214-218.

29. Zarzar, L. D.; Liu, Q.; He, X.; Hu, Y.; Suo, Z.; Aizenberg, J. Multifunctional Actuation Systems Responding to Chemical Gradients. Soft Matter 2012, 8, 8289-8293.30. He, X.; Friedlander, R. S.; Zarzar, L. D.; Aizenberg, J. Chemo-mechanically Regulated Oscillation of an Enzymatic Reaction. Chem. Mater. 2013, 25, 521-523.

31. Kim, P.; Zarzar, L. D.; He, X.; Grinthal, A.; Aizenberg, J. Hydrogel-Actuated Integrated Responsive Systems (HAIRS): Moving towards Adaptive Materials. Curr. Opin. Solid State Mater. Sci. 2011, 15, 236-245.

32. Pokroy, B.; Epstein, A. K.; Persson-Gulda, M. C. M.; Aizenberg, J. Fabrication of Bioinspired Actuated Nanostructures with Arbitrary Geometry and Stiffness. Adv. Mater. 2009, 21, 463469.31. 
33. Kim, P.; Zarzar, L. D.; Khan, M.; Aizenberg, M.; Aizenberg, J. Environmentally Responsive Active Optics based on Hydrogel-Actuated Deformable Mirror Arrays. Proc. SPIE 2011, 7927, 792705.

34. Matsunaga, M.; Aizenberg, M.; Aizenberg, J. Controlling the Stability and Reversibility of Micropillar Assembly by Surface Chemistry. J. Am. Chem. Soc. 2011, 133, 5545-5553.35.

Hatton, B. D.; Aizenberg, J. Writing on Superhydrophobic Nanopost Arrays: Topographic Design for Bottom-up Assembly. Nano Lett. 2012, 12, 4551-4557.33.

36. Hong, W.; Zhao, X.; Suo, Z. Drying-induced Bifurcation in a Hydrogel-Actuated Nanostructure. J. Appl. Phys. 2008, 104, 084905.37. Tanaka, T.; Sun, S-T.; Hirokawa, Y.; Katayama, S.; Kucera, J.; Hirose, Y, Amiya, T. Mechanical Instability of Gels at the Phase Transition. Nature 1987, 325, 796-798. 